\title{
Спектры электроотражения множественных квантовых ям InGaN/GaN, помещенных в неоднородное электрическое поле $p-n$-перехода
}

\author{
(С) Л.П. Авакянц, А.Э. Асланян, П.Ю. Боков , К.Ю. Положенцев, А.В. Червяков \\ Московский государственный университет им. М.В. Ломоносова (физический фракультет), \\ 119991 Москва, Россия \\ ฯ E-mail: pavel_bokov@physics.msu.ru
}

(Получена 12 апреля 2016 г. Принята к печати 20 апреля 2016 г.)

В спектрах электроотражения гетероструктуры $\mathrm{GaN} / \mathrm{InGaN} / \mathrm{AlGaN}$ обнаружена линия $E=2.77$ эВ шириной $\Gamma=88$ мэВ, связанная с межзонными переходами в области множественных квантовых ям активной области. При уменьшении амплитуды модулирующего напряжения от 2.9 до 0.4 В наблюдается расщепление этой линии на две с энергиями $E_{1}=2.55$ эВ и $E_{2}=2.75$ эВ, ширины которых составляют $\Gamma_{1}=66$ мэВ и $\Gamma_{2}=74$ мэВ соответственно. Это указывает на то, что эти линии обусловлены межзонными переходами в отдельных квантовых ямах активной области. Различие энергий межзонных переходов $E_{1}$ и $E_{2}$ в идентичных квантовых ямах активной области связано с тем, что квантовые ямы помещены в неоднородное электрическое поле. В работе оценивались модули напряженности электрических полей в отдельных квантовых ямах активной области гетероструктуры. Их значения составили 1.6 и $2.2 \mathrm{MB} / \mathrm{cm}$.

DOI: 10.21883/FTP.2017.02.44104.8271

\section{1. Введение}

Излучающей частью современных светодиодных гетероструктур является помещенная в $p-n$-переходе активная область, которая содержит одну или несколько квантовых ям [1]. Квантовые ямы аккумулируют инжектируемые через $p-n$-переход носители заряда, увеличивая вероятность излучательной рекомбинации в активной области. Увеличение числа квантовых ям необходимо для уменьшения концентрации носителей в отдельных ямах, так как их переизбыток приводит к росту вероятности оже-рекомбинации ([1], с. 56; [2]). Пространственное разделение электронов и дырок в квантовой яме, обусловленное наличием пьезоэлектрических полей, уменьшает вероятность излучательной рекомбинации $[3,4]$. В работе [5] показано, что в системе множественных квантовых ям (МКЯ) основную роль в формировании излучения, как правило, играет одна квантовая яма. Поэтому исследование неоднородности встроенных пьезоэлектрических полей в квантовых ямах активной области светодиодной гетероструктуры является актуальным.

Светодиоды, изготовленные на сапфировой подложке и смонтированные на теплоотвод по технологии „flip-chip“, допускают исследования методами спектроскопии отражения [4], фотоотражения [6] и электроотражения (ЭО) [7]. В последнем случае модуляция отражения осуществляется приложением к контактам $p-n$-перехода переменного напряжения. Прикладывая к контактам $p$-n-перехода переменное напряжение с разной постоянной составляющей, можно оценивать напряженности пьезоэлектрических полей за счет эффекта Штарка [8].

В данной работе метод спектроскопии ЭО применен для исследования неоднородности встроенных пьезоэлектрических полей в квантовых ямах активной области светодиодной гетероструктуры GaN/InGaN/AlGaN.
Обнаружено, что при малых амплитудах модулирующего напряжения, линия, связанная с сигналом от МКЯ, расщепляется на две. Указанное расщепление спектральных линий происходит за счет уменьшения их перекрытия. По разнице энергий компонент расщепленной линии оценены значения напряженностей электрических полей в отдельных квантовых ямах активной области гетероструктуры.

\section{2. Образец и методика эксперимента}

Исследованный образец представлял собой светодиод сине-зеленого свечения. Гетероструктура на основе МКЯ типа GaN/InGaN/AlGaN выращивалась методом газофазной эпитаксии из металлоорганических соединений (MOCVD) на сапфировой подложке и монтировалась в теплоотводящий корпус методом „flip-chip“ [9]. На подложке последовательно выращивались (рис. 1, $a$ ): затравочный слой $\mathrm{GaN}$ толщиной 20 нм; буферный слой $i-\mathrm{GaN}$ толщиной < 0.7 мкм; слой $n$-GaN толщиной 4.5 мкм; буферная сверхрешетка InGaN/GaN; активная область - 5 квантовых ям/барьеров $\operatorname{In}_{x} \mathrm{Ga}_{1-x} \mathrm{~N} / \mathrm{GaN}$ толщиной 4 нм/4 нм; слой, блокирующий поперечный транспорт электронов $p-\mathrm{Al}_{x} \mathrm{Ga}_{1-x} \mathrm{~N}$ (20 нм), $x=0.12-0.15$ ([1], с. 107); слой $p$-GaN толщиной 0.12 мкм. Со стороны контакта к p-области монтировалось отражающее зеркало. Излучение такого светодиода, формируемое в активной области, отражается от зеркала-теплоотвода и выводится наружу через прозрачную в сине-зеленой части спектра сапфировую подложку. Площадь кристалла составляла $0.4 \mathrm{Mм}^{2}$.

Измерение спектров ЭО проводилось на установке, описанной ранее в работах $[4,6]$. Вторая часть монохроматора в ходе измерений не задействовалась: анализировалось отраженное от образца монохроматиче- 


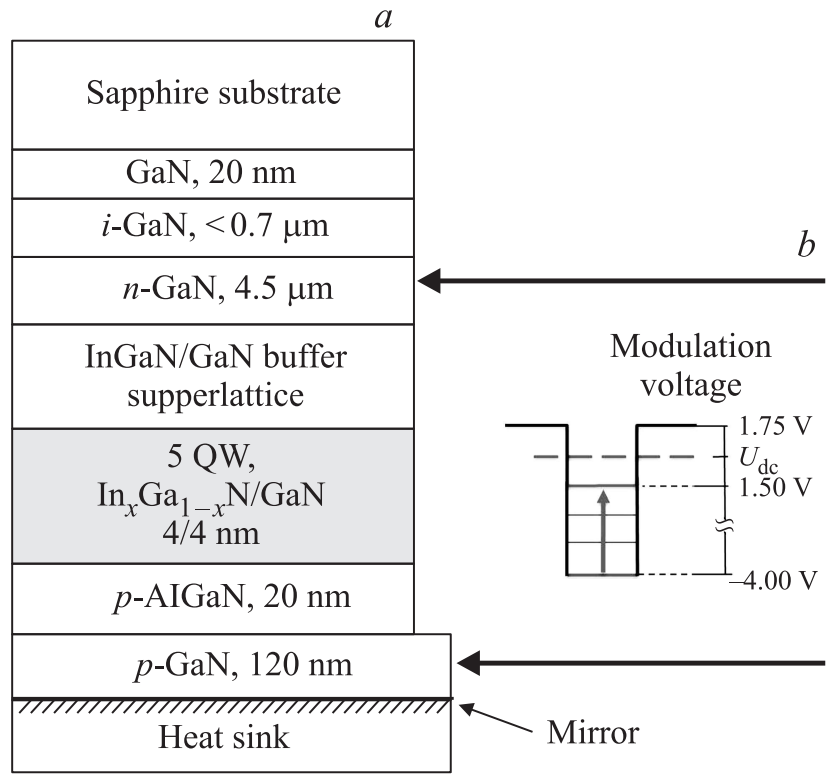

Рис. 1. Структура исследуемого светодиода (a) и схема приложения к $p-n$-переходу модулирующего напряжения $(b)$.

ское излучение, сформированное первым монохроматором. Гетероструктуры GaN/InGaN/AlGaN освещались через сапфировую подложку (рис. 1,a). Спектральный диапазон падающего на образец излучения составлял $390-500$ нм. Угол падения составлял $8^{\circ}$.

Для модуляции коэффициента отражения $R$ к контактам $p-n$-перехода прикладывались импульсы напряжения прямоугольной формы. Частота модуляции составляла 370 Гц. В серии экспериментов амплитуда импульсов $U_{\bmod }$ и постоянное смещение $U_{\mathrm{dc}}$ (рис. $\left.1, b\right)$ изменялись независимо. Постоянное смещение $U_{\mathrm{dc}}$ выбиралось из диапазона, обеспечивающего запирание $p-n$-перехода. Максимальное значение напряжения модулирующего меандра, равное $1.75 \mathrm{~B}$, не менялось, а минимальное изменялось в пределах от -4.00 до $1.00 \mathrm{~B}$. Спектры ЭО регистрировались при комнатной температуре. Указанному режиму соответствуют изменения постоянного смещения $U_{\mathrm{dc}}$ от -1.1 до $1.7 \mathrm{~B}$ и амплитуды сигнала $U_{\text {mod }}$ от 0.1 до $2.9 \mathrm{~B}$.

Таким образом, сигнал ЭО $\Delta R / R$ может быть описан как

$$
\frac{\Delta R}{R}=\frac{R\left(U_{\mathrm{mod}}+U_{\mathrm{dc}}\right)-R\left(U_{\mathrm{dc}}\right)}{R}=\frac{R\left(F_{1}\right)-R\left(F_{2}\right)}{R},
$$

где $F_{1}$ и $F_{2}$ - напряженности электрического поля в структуре (в квантовой яме) при приложении модулирующего напряжения $U_{\bmod }$ и в его отсутствии. В выбранном режиме модуляции все падение напряжения приходится на область запертого $p-n$-перехода, при этом увеличение обратного смещения приводит к уменьшению напряженности электрического поля в квантовых ямах InGaN/GaN активной области [4], а уменьшение амплитуды модулирующего напряжения приводит к меньшим изменениям напряженности электрического поля $F_{1}-F_{2}$.

\section{3. Результаты и их обсуждение}

На рис. 2 приведены спектры ЭО, зарегистрированные при указанных амплитудах модулирующего напряжения $U_{\text {mod. }}$ В спектрах присутствуют линии в области энергий (2.63-3.0) эВ, соответствующие межзонным переходам в МКЯ активной области гетероструктуры [10]. Видно, что при напряжениях $\left(1.9 \leq U_{\bmod } \leq 2.9\right)$ В наблюдается единая широкая спектральная линия $\left(, E^{6}\right)$, амплитуда которой уменьшается с уменьшением $U_{\text {mod }}$. При $U_{\mathrm{mod}} \leq 1.4 \mathrm{~B}$ возникает расщепление этой линии на две, обозначенные на рис. 2 как „, $E_{1}$ “ и , $E_{2}$ “. Линия „, $E_{1}$ “ в области меньших энергий имеет бо́льшую амплитуду,

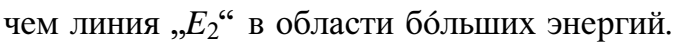

Широкий контур линии в спектре ЭО представляет собой совокупность линий от тех квантовых ям активной области, в которых электрическое поле модулируется наиболее интенсивно. Наблюдаемое расщепление линии связано одновременно с уменьшением перекрытия отдельных линий, ширина каждой из которых уменьшается с уменьшением $U_{\text {mod }}$ и со сдвигами линий „ $E_{1}$ “ и „, $E_{2}{ }^{“}$. Последнее можно объяснить неоднородностью электрического поля в $p-n$-переходе, приводящего к сдвигам энергетических уровней электронов и дырок в силу эффекта Штарка. Различие амплитуд линий „E $E_{1}$ “ и „E $E_{2}$ “ можно объяснить различной глубиной модуляции

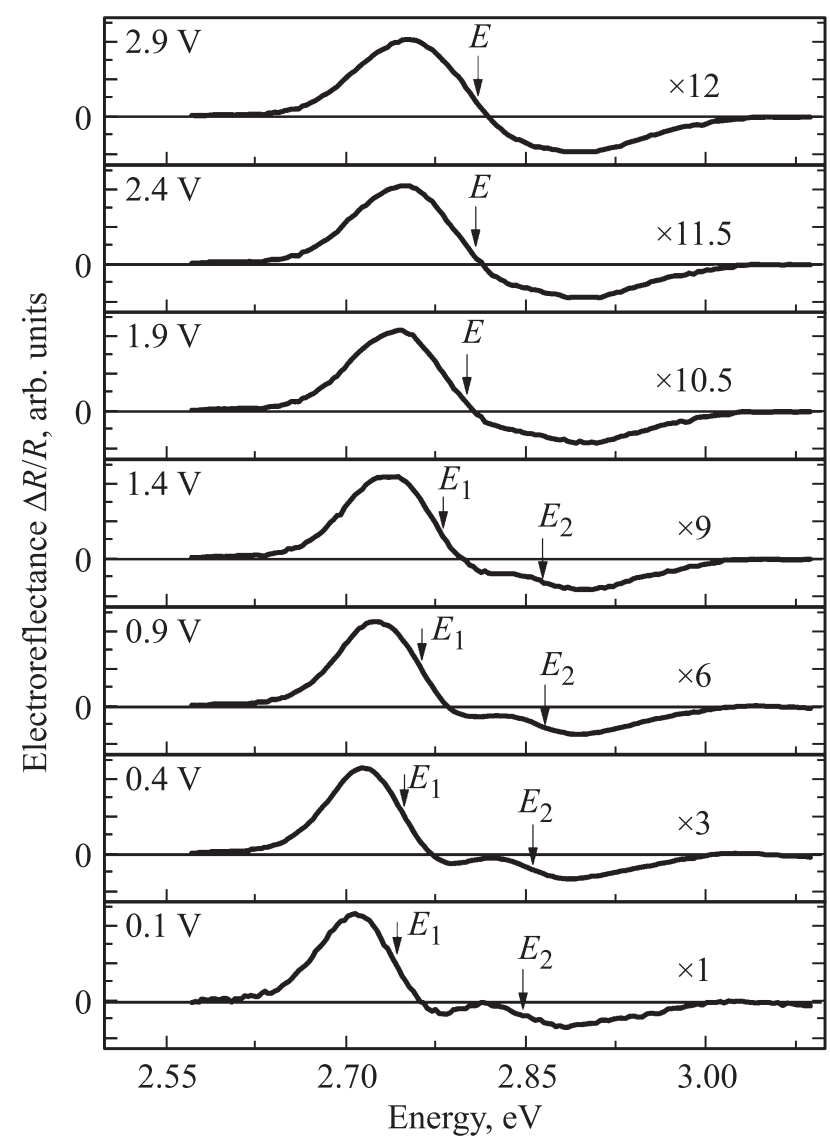

Рис. 2. Спектры ЭО, полученные при амплитудах модулирующего напряжения $U_{\text {mod }}$ от 0.1 до $2.0 \mathrm{~B}$. 
электрического поля в квантовых ямах, ответственных за формирование соответствующих линий в спектре ЭО.

Определение параметров единой широкой спектральной линии, т.е. полученной при больших амплитудах модулирующего сигнала, проводилось с помощью выражения Аспнеса [11]:

$$
\frac{\Delta R}{R}=\operatorname{Re}\left(A \cdot e^{i \varphi}\left(E-E_{i}+i \Gamma\right)^{-m}\right)
$$

где $A, \varphi$ - амплитудный и фазовый параметры соответственно, $E=\hbar \omega-$ энергия отраженного излучения, $E_{i}$ - энергия перехода, соответствующая спектральной линии, Г - феноменологический параметр уширения, $m$ - размерность критической точки (в нашем случае

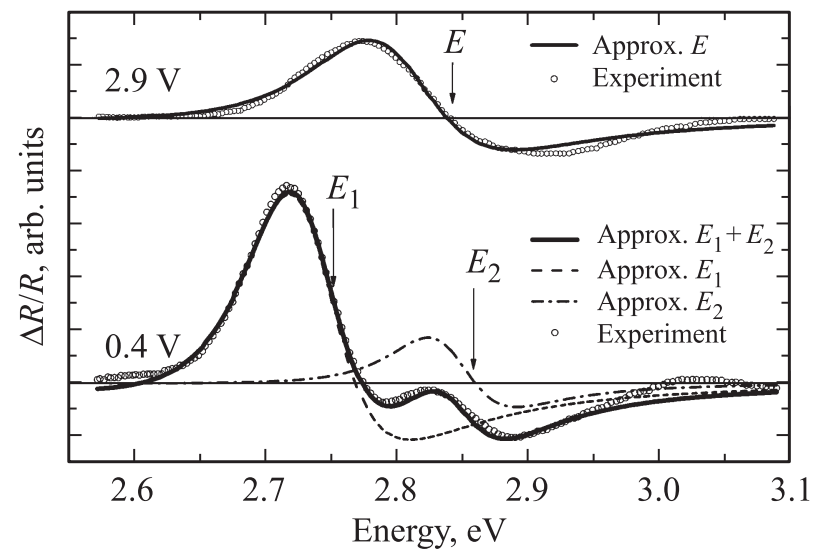

Рис. 3. Пример аппроксимации спектров ЭО, соответствующих двум амплитудам модуляции $U_{\text {mod: }} 2.9$ и 0.4 В. Штриховая и штрихпунктирная линии - аппроксимация линий $E_{1}$ и $E_{2}$, сплошные линии - суммарная апрроксимация двух выражений Аспнеса для $U_{\mathrm{mod}}=0.4 \mathrm{~B}$ и аппроксимация по формуле (1) для $U_{\mathrm{mod}}=2.9 \mathrm{~B}$.

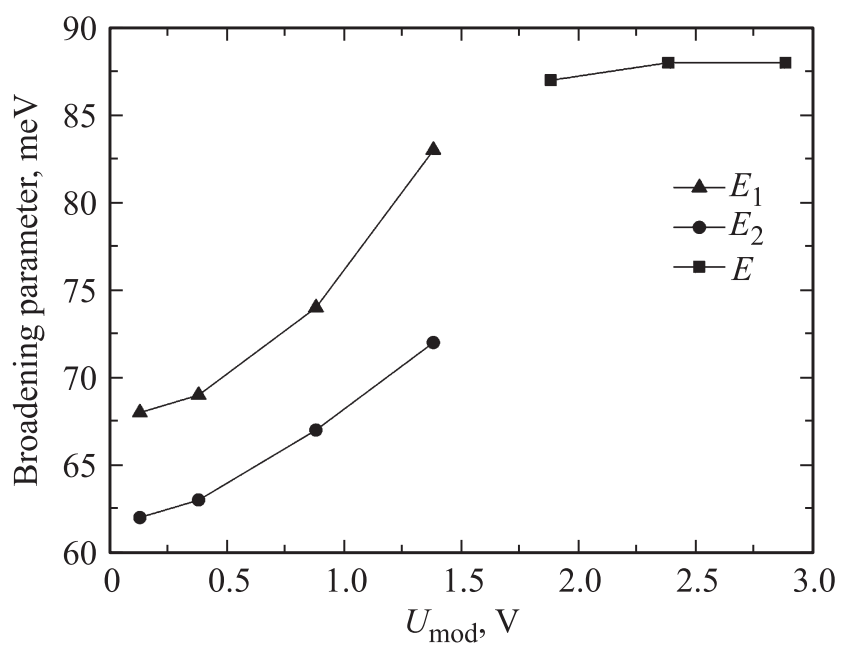

Рис. 4. Зависимость параметра уширения линий спектров ЭО от амплитуды модулирующего сигнала. Квадратами отмечены точки, полученные аппроксимацией по формуле (1) для единой спектральной линии. Треугольниками и квадратами отмечены точки, полученные аппроксимацией суммой двух выражений Аспнеса (1) для линий $E_{1}$ и $E_{2}$.

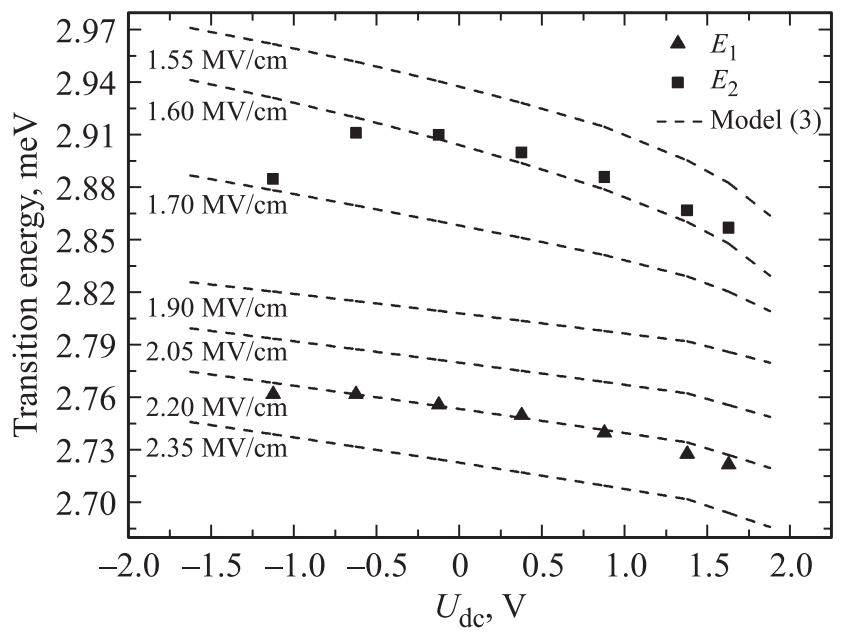

Рис. 5. Зависимость энергий переходов $E_{1}$ (треугольники) и $E_{2}$ (квадраты) в квантовых ямах активной области от напряжения смещения. Штриховые линии - теоретические зависимости (2), соответствующие различным значениям напряженности электрического поля $F$.

$m=2[11])$. Для аппроксимации спектров с расщепленными линиями (при $U_{\text {mod }} \leq 1.3 \mathrm{~B}$ ) в качестве подгоночной формулы использовалась сумма двух выражений (1) Аспнеса (рис. 3).

На рис. 4 приведен график зависимости параметра уширения от амплитуды модулирующего напряжения. В диапазоне напряжений $\left(1.9 \leq U_{\bmod } \leq 2.9\right)$ В это одна широкая линия с $\Gamma \approx 88$ эВ. В диапазоне напряжений $\left(0.1 \leq U_{\bmod } \leq 1.4\right)$ В это две линии с монотонно уменьшающимися параметрами уширения при уменьшении $U_{\bmod }\left(\Gamma_{1}\right.$ от 83 до 66 мэВ; $\Gamma_{2}$ от 72 до 63 мэВ).

Определенные по результатам аппроксимации зависимости энергий переходов в квантовых ямах $E_{1}$ и $E_{2}$ от модулирующего напряжения $U_{\text {mod }}$ представлены на рис. 5. Видно, что увеличение $U_{\bmod }$ приводит к сдвигу обеих линий в область бо́льших энергий. Наблюдаемые уменьшения параметров уширения и сдвиги линий в область меньших энергий при уменьшении амплитуды модулирующего напряжения указывают на то, что основным механизмом, ответственным за формирование линий в спектрах ЭО, является квантово-размерный эффект Штарка. При этом с ростом амплитуды модулирующего напряжения неоднородности электрического поля в области множественных квантовых ям частично маскируются электрическим полем, формируемым при модуляции. Последнее и приводит к уширению и перекрытию линий ЭО.

В работе [12] предложено выражение, описывающее зависимость значения энергии $E_{1 e 1 h}$ квантовой ямы светодиодной гетероструктуры от напряженности электрического поля $F$ в квантовой яме:

$$
E_{1 e 1 h}=E_{1 e 1 h}(F=0)-\frac{15-\pi^{2}}{24 \pi^{4}} \frac{\left(m_{e}+m_{h}\right) e^{2} F^{2} L_{w}^{4}}{\hbar^{2}},
$$

где $E_{1 e 1 h}-$ энергия электронно-дырочного перехода в присутствии электрического поля $F, E_{1 e 1 h}(F=0)-$ 
энергия электронно-дырочного перехода в отсутствии электрического поля в квантовой яме, $m_{e}, m_{h}$ - эффективные массы электронов и дырок, $F$ - модуль вектора напряженности внутреннего электрического поля, $L_{w}-$ ширина квантовой ямы.

Видно (см. рис. 5), что экспериментальные значения $E_{1}$ сосредоточены вблизи линии, соответствующей напряженности электрического поля $F_{1}=1.60 \mathrm{MB} / \mathrm{cM}$, а $E_{2}$ - соответствующей напряженности электрического поля $F_{2}=2.20 \mathrm{MB} / \mathrm{cm}$.

Эти данные согласуются с расчетом модуля встроенного электрического поля в рамках теории пьезоэлектрического эффекта для такого рода структур: $1.9 \pm 0.3 \mathrm{MB} / \mathrm{cm}[13]$ и нашими предыдущими экспериментами [14]. Таким образом, паре квантовых ям, дающих основной вклад в спектр ЭО, соответствуют электрические поля, напряженности которых различаются на $0.6 \mathrm{MB} / \mathrm{cm}$. Относительная неоднородность электрического поля составляет $32 \%$.

\section{4. Заключение}

При комнатной температуре зарегистрированы спектры ЭО светодиодной гетероструктуры на основе соединений $\mathrm{GaN} / \mathrm{InGaN} / \mathrm{AlGaN}$ при амплитудах модулирующего напряжения от 0.1 до $2.9 \mathrm{~B}$. В спектрах ЭО присутствует линия, связанная с межзонными переходами $1 e-1 h$ в области МКЯ. Наблюдаемые уменышения параметров уширения и сдвиги линий в область меньших энергий при уменьшении амплитуды модулирующего напряжения указывают на то, что основным механизмом, ответственным за формирование линий в спектрах ЭО, является квантово-размерный эффект Штарка.

Различие в энергиях линий ЭО, наблюдаемых от области МКЯ, позволяет сделать вывод о том, что электрическое поле в активной области $p-n$-перехода неоднородно.

Исследована зависимость энергии переходов в квантовых ямах от приложенного напряжения к $p-n$-переходу. По зависимости энергий переходов для двух спектральных линий от прикладываемого к контактам $p-n$-перехода напряжения оценены значения модуля напряженности электрических полей отдельных квантовых ям активной области. Для первой квантовой ямы эта оценка составила $1.60 \mathrm{MB} / \mathrm{cm}$, для второй $-2.20 \mathrm{MB} / \mathrm{cm}$.

Показано, что метод спектроскопии ЭО может использоваться не только для оценки напряженности электрического поля в активной области $p-n$-перехода светодиодной полупроводниковой гетероструктуры, но и для оценки его неоднородности.

\section{Список литературы}

[1] Ф. Шуберт. Светодиоды (М., Физматлит, 2008).

[2] N.F. Gardner, G.O. Mueller, Y.C. Shen, G. Chen, S. Watanabe, W. Gotz, M. R. Krames. Appl. Phys. Lett., 91, 243506 (2007).
[3] H. Zhao, L. Guangyu, J. Zhang, J.D. Poplawsky, V. Dierolf, N. Tans. Opt. Express, 19 (S4), 991 (2011).

[4] Л.П. Авакянц, М.Л. Бадгутдинов, П.Ю. Боков, А.В. Червяков, С.С.Широков, А.Э. Юнович, А.А. Богданов, Е.Д. Васильева, Д.А. Николаев, А.В. Феопентов. ФТП, 41 (9), 1078 (2007).

[5] A. David, M.J. Grundmann, J.F. Kaeding, N.F. Gardner, T.G. Mihopoulos, M.R. Krames. Appl.Phys. Lett., 92, 053502 (2008).

[6] Л.П. Авакянц, П.Ю. Боков, А.В. Червяков. ЖТФ, 75 (10), 66 (2005).

[7] R.J. Kaplar, S.R. Kurtz, D.D, Koleske, A.J. Fischer. Appl. Phys. Lett., 95, 4905 (2004).

[8] T. Takeuchi, C. Wetzel, S. Yamaguchi, H. Sakai, H. Amano, I. Akasaki, Y. Kaneko, S. Nakagawa, Y. Yamaoka, N. Yamada. Appl. Phys. Lett., 73, 1691 (1998).

[9] J.J. Wierer, D.A. Steigerwald, M.R. Krames, J.J. O’Shea, M.J. Ludowise, G. Christenson, Y.-C. Shen, C. Lowery, P.S. Martin, S. Subramanya, W. Götz, N.F. Gardner, R.S. Kern, S.A. Stockman. Appl. Phys. Lett., 78 (22), 3379 (2001).

[10] H. Shen, M. Dutta. J. Appl. Phys., 78, 2151 (1995).

[11] D. Aspnes. Surf. Sci., 37, 418 (1973).

[12] M.E. Aumer, S.F. Le Boeuf, B.F. Moody, S.M. Bedair. Appl. Phys. Lett., 79 (23), 3803 (2001).

[13] M. Feneberg, K. Thonke. J. Phys.: Condens. Matter, 19, 403201 (2007).

[14] L.P. Avakyants, P.Yu. Bokov, A.V. Chervyakov, A.E. Yunovich, E.D. Vasileva, B.S. Yavich. Phys. Status Solidi C, 7, 1863 (2010).

Редактор А.Н. Смирнов

\section{Electroreflectance spectra from InGaN/GaN multiple quantum wells placed into non-uniform electric field of $\boldsymbol{p}-\boldsymbol{n}$-junction}

\author{
L.P. Avakyants, A.E. Aslanyan, P.Yu. Bokov, \\ K.Yu. Polozhentsev, A.V. Chervyakov \\ Lomonosov Moscow State University \\ (Faculty of Physics), \\ 119991 Moscow, Russia
}

\begin{abstract}
There was spectral line at $E=2.77 \mathrm{eV}$ with broadening $\Gamma=88 \mathrm{meV}$ in electroreflectance spectra of heterostructure based on $\mathrm{GaN} / \mathrm{InGaN} / \mathrm{AlGaN}$ connected with interband transition in area of quantum wells. Reduction of the amplitude of modulation bias from 2.9 to $0.4 \mathrm{~V}$ leads to splitting this line into two with transition energies of $E_{1}=2.55 \mathrm{eV}$ and $E_{2}=2.75 \mathrm{eV}$ with broadening $\Gamma_{1}=66 \mathrm{meV}$ and $\Gamma_{2}=74 \mathrm{meV}$. The smaller width of the separate lines points that these lines were provided by interband transitions in particular quantum wells of active area. The difference between interband transition energy $E_{1}$ and $E_{2}$ into similar quantum wells reveal that these quantum wells put into inhomogeneous electric field of $p-n$-junction. The magnitudes of electric fields strengths in particular quantum wells in active area of the heterostructure have been estimated as 1.6 and $2.2 \mathrm{MV} / \mathrm{cm}$.
\end{abstract}

